understand the use of QRISK-3 in an ID clinic and to quantify individual CVD risks to recommend appropriate management options. Method. A cross sectional study was performed on 143 patients open to an ID psychiatry clinic. Patients and carers were sent an accessible information leaflet on this study. Basic demographic data and information on psychiatric diagnoses were collected. Patients were grouped according to the presence of severe mental illness (SMI) defined as schizophrenia, bipolar disorder and other psychotic illnesses. QRISK-3 $\geq 10 \%$ was defined as elevated risk in accordance with NICE guidelines. Patients who had a high QRISK-3 score were advised to contact their GP.

Result. Of 143 patients, $73(51.0 \%)$ had a mild ID and the remaining had a moderate to severe ID. The mean age was 43.3 years, 53.1\% were male. Overall, 28 (19.6\%) participants had an elevated CVD risk, of whom 16 (57.1\%) were not on statins, which is the recommended treatment. The mean QRISK-3 score was 6.31 (standard deviation [SD] 8.95), and the relative risk is 3.50 (SD 7.13). The proportion of QRISK- $3 \geq 10 \%$ and mean score were not significantly different in those with SMI, but those with SMI were more likely to be prescribed statins than those without $(14$ [31.1\%] vs 10 [10.2\%], p=0.002). Statins were given to $24(16.8 \%)$ participants, of whom 12 (50\%) had elevated CVD risk. $89 \%$ had a blood pressure recording within the past 5 years, $87 \%$ had height and $88 \%$ had weight recorded. $73 \%$ had lipid serology results recorded.

Conclusion. Elevated CVD risk was common in this ID study population, and more than half with elevated QRISK-3 were not on the medical treatment recommended by national guidelines. QRISK-3 could feasibly be implemented in the outpatient setting. Increased routine CVD risk assessment and management should be considered as another measure to reduce morbidity and mortality.

\section{A case of olanzapine-associated rhabdomyolysis}

Valentin Skryabin $^{1 \star}$, Michael Zastrozhin ${ }^{1}$, Dmitry Sychev ${ }^{2}$ and Evgeny Bryun ${ }^{3}$

${ }^{1}$ Moscow Research and Practical Centre on Addictions of the Moscow Department of Healthcare, Russian Medical Academy of Continuous Professional Education of the Ministry of Health of the Russian Federation; ${ }^{2}$ Russian Medical Academy of Continuous Professional Education of the Ministry of Health of the Russian Federation and ${ }^{3}$ Moscow Research and Practical Centre on Addictions of the Moscow Department of Healthcare

${ }^{\star}$ Corresponding author.

doi: $10.1192 /$ bjo.2021.188

Aims. To describe the case of olanzapine-associated rhabdomyolysis in a 20 -year-old patient with a suspected diagnosis of paranoid schizophrenia.

Method. A 20-year-old male Caucasian patient was admitted to the Psychiatric Department with a one-month history of irrational behavior, talking to himself, persecutory delusions, and poor sleep. He was prescribed oral olanzapine at a dose of $10 \mathrm{mg}$ per day. After two days of olanzapine monotherapy, the patient experienced muscle jerks in the legs. Four days after the initiation of olanzapine treatment, he complained about fatigue and weakness in the lower extremities along with myalgia. Physical examination revealed decreased muscle power with no extrapyramidal symptoms. Blood chemistry showed serum creatine kinase $(\mathrm{CK})$ and serum lactate dehydrogenase (LDH) of 9,725 U/L and $843 \mathrm{U} / \mathrm{L}$, respectively, on day four of the therapy. The Naranjo algorithm score of 6 suggested that olanzapine was the probable cause of rhabdomyolysis. A diagnosis of drug-induced rhabdomyolysis was established from the background of blood tests (increased serum CK and LDH levels), clinical presentation (fatigue and weakness in the lower extremities, muscle jerks, and myalgia), and Naranjo algorithm score of 6 for olanzapine. On suspicion of its contribution to rhabdomyolysis, olanzapine was immediately withdrawn. The patient was referred to the intensive care unit. To prevent acute renal failure, high-volume alkaline diuresis was initiated. After consulting a clinical pharmacologist, the patient's primary physician decided to perform a pharmacogenetic test to develop an individualized treatment regimen. Pharmacogenetic test results were interpreted using the PGX2 software (Meditsina LLC, Moscow, Russia). The test revealed that the patient was a homozygous mutant for CYP2D6*4, which corresponds to CYP2D6 PM phenotype. With this in mind, trifluoperazine was prescribed at a daily dose of $10 \mathrm{mg}$ instead of olanzapine as recent data indicate that trifluoperazine is metabolized by CYP1A2 and UGT1A4 instead of CYP2D6. Subsequently, the patient recovered well and was discharged without any nephrological sequelae.

Result. Recent research demonstrates that CYP2D6 is one of the most important isoenzymes implicated in drug metabolism because the CYP2D6 gene is highly polymorphic. Few reports on the association between olanzapine use and rhabdomyolysis have been published to date, and the present case report draws attention to pharmacogenetic testing which allowed the psychiatrist to prescribe another antipsychotic with no risk of rhabdomyolysis.

Conclusion. The presented case demonstrates that pharmacogenetic-guided personalization of treatment may allow selecting the best medication and determining the right dosage, resulting in the reduced risk of adverse drug reactions and pharmacoresistance.

Effects of tailored quality improvement programme for effective medication management in high dependency in-patient psychiatry rehabilitation unit

Hina Tahseen* and Jade Brown

Delfryn House, Cygnet Health Care

${ }^{*}$ Corresponding author.

doi: 10.1192/bjo.2021.190

Aims. To determine the effects of a tailored quality improvement programme for effective medication management including a reduction in prescription and administration errors in oral and depot psychotropic medication, patient education on medication and implementation of policies and guidelines.

Background. Medication errors are common in hospital admissions and pose a threat to patient safety (Buckley et al. 2013). Medication errors may occur in different stages of the patient treatment process such as during prescribing, transcribing, preparing, dispensing, administration, and monitoring (Wang et al. 2015). In addition to these, for the detained mental health patients, the Mental Health Act 1983 legislation requires up-to-date treatment certificate compliance (Wales. Welsh Assembly 2008). A Quality Improvement programme to improve safe medication prescription and administration was designed for the patients admitted in Delfryn House, a mental health high dependency rehabilitation unit.

Method. Using Plan-Do-Study-Act (PDSA) quality improvement methodology, a medication management committee was created under the leadership of Specialty doctor and Head of Care (HOC), and comprising of the consultant psychiatrists, specialty doctor, heads of care (ward managers), senior nurses, 
pharmacists, hospital manager and hospital director. The committee reviewed the medication errors reported in the last year and planned the Pre-Intervention Phase 1 and Post Intervention Phase II Audits.

The Intervention project was broadly divided into two domains---Doctors' Prescription led by the Specialty doctor and the Nurses' Medication Administration, led by the Head of care. Using the QI "theory of change" model, three primary drivers of "Safe Prescription and Administration", "Patient Education" and "Policies and Guidelines Implementation" were established. The poster will have a demonstration of the complete drivers' diagram.

Secondary drivers for "Safe prescription and administration" required inputs from doctors, nurses and pharmacists; Change ideas (Interventions) of introducing In-patient depot clinics, Daily 10-Points self-audit by clinic nurse, twice daily information about patients' medication compliance in morning and evening electronic handovers, PDSAs with monthly audits of prescription and administration errors, monthly pharmacists' audits for drug interactions and monitoring of adverse effects and rapid tranquilisations were implemented.

Secondary drivers and change ideas for "Patient Education" included discussions with Multidisciplinary teams, medication information leaflets being available to patients, discussion slots with pharmacists, self-administration of medication, and alternate self-management strategies instead of PRN medications.

Secondary drivers and change ideas for the "Policies and Guidelines Implementation" included steps to ensure all staff were aware of the policies for safe drug administration, rapid tranquilization and PRN utilisation, medication meetings minutes being circulated to all staff, and monthly audits for MHA1983 Section 57 treatment certificates for detained patients.

The medication Management Committee continued to meet on monthly basis to review the interventions, implementation of new strategies, and new recommendations on the basis of monthly mini-audits. A patient satisfaction survey on their knowledge about prescribed psychotropic medication was also conducted pre and post-intervention.

Result. Results of Phase I and Phase 11 were compared. There was a significant reduction in prescription errors by doctors (19\% to $3 \%$ ) and medication administration (34\% to $11 \%)$. Mental health documentation compliance improved from $77 \%$ to $98 \%$. Patient satisfaction survey also demonstrated more knowledge about their prescribed psychotropic medication (15\% to $32 \%)$. Two areas however did not show satisfactory improvements; There was not a significant improvement in acknowledgment or documentation of potential drug interactions or adverse events raised by pharmacists. Errors related to depot medication administration reduced in the initial two months, but increased again. The introduction of the Weekly Depot Clinic was not found successful by the administering nursing staff, and it was moved back to daily administrations.

Conclusion. The formation of the medication management committee and the quality improvement programme showed significant improvement in most areas of effective medication management. The primary and secondary drivers with the change ideas gave structure to the intervention programme. The mini-audits using PDSA methodology helped to test different interventional strategies and to assess their impact and building upon the learning from previous results. This shows that for sustained effective medication management, this should not be a one-off exercise, and we need to continue learning and implementing newer strategies for continued effective medication, taking on-board the advice from MDT, nursing, patients, and carers.

\section{References}

Buckley, M. S. et al. 2013. Impact of a Clinical Pharmacy Admission Medication Reconciliation Program on Medication Errors in "High-Risk" Patients. The Annals of pharmacotherapy 47(12), pp. 1599-1610. doi: $10.1177 / 1060028013507428$

Wales. Welsh Assembly, G. 2008. Mental Health Act 1983 : Code of Practice for Wales. Cardiff]: Cardiff : Welsh Assemby Government.

Wang, H.-F. et al. 2015. Quality improvements in decreasing medication administration errors made by nursing staff in an academic medical center hospital: a trend analysis during the journey to Joint Commission International accreditation and in the post-accreditation era. Therapeutics and clinical risk management 11, pp. 393-406. doi: 10.2147/TCRM.S79238

\section{Improving remote prescribing in a CAMHS community team during the COVID-19 pandemic}

Sarah Tai*, Hannah Chu-Han Huang, Oliver Batham, Brindha Anandakumar and Christopher Abbott

South London and Maudsley NHS Foundation Trust ${ }^{\star}$ Corresponding author.

doi: 10.1192/bjo.2021.191

Aims. Prior to the COVID-19 pandemic, prescriptions were usually collected by patients/families in person from the CAMHS community team base. Due to social distancing measures introduced during the pandemic, face-to-face contact between staff and patients had to be minimised. This led to an increase in remote prescribing, including from home. Feedback from team doctors was that the process of following the Remote Prescribing Protocol (RPP) was taking up a significant portion of their day, preventing them from doing other clinical work.

Our aim was to reduce the time taken to complete a remote prescription to pre-pandemic levels (under 15 minutes).

Method. We used PDSA methodology in this QI project:

1) Plan: Survey sent out to team duty doctors to identify the most time-consuming steps in RPP which could be safely delegated to administrative staff

2) Do: Email sent requesting administrative staff clarify several details with patients/families when they request a prescription. This included the names and doses of medication, how many days they had left, where they wanted the prescription sent to (home/pharmacy) and the relevant address. If the patient usually received their repeat prescription from their GP, they were re-directed to their GP

3) Study: Following the intervention above, team doctors recorded how long it took to complete a remote prescription

Result. The average time taken to complete a prescription fell from 31 minutes (pre-intervention) to 22 minutes (postintervention). The range of time taken also dropped from 10-241 minutes (pre-intervention) to 0-46 minutes (postintervention). The medications taking above the average time to complete were more likely to be non-controlled drugs rather than controlled drugs (which one may typically think would be more time-consuming to write out).

Conclusion. Whilst we have successfully reduced the time for remote prescribing, we have not reached the target of reducing it down to less than 15 minutes (pre-pandemic timings). As part of the next PDSA cycle, we have carried out a survey to ask what barriers remain. Checking patient's notes and recent prescriptions can still be inefficient. We propose introducing an intervention whereby this can also be safety delegated to administrative staff e.g. including a copy of the most recent prescription in the request. 